\section{High lymphoid enhancer- binding factor-1 expression is associated with disease progression and poor prognosis in chronic lymphocytic leukemia}

\section{Felix Erdfelder, Magdalena Hertweck, Alexandra Filipovich, Sabrina Uhrmacher, Karl-Anton Kreuzer}

Department I of Internal Medicine, University at Cologne, Germany

\begin{abstract}
We determined lymphoid enhancer-binding factor-1 (LEF1) mRNA expression in 112 chronic lymphocytic leukemia (CLL) samples and assessed correlations with the prognostic markers ZAP70 and CD38, Binet stages, the percentage of lymphocytes in the peripheral blood, and fibromodulin (FMOD) transcripts. The mean LEF1 relative expression ratios (RER) were 53.72 and 37.10 in ZAP70-positive and ZAP70 negative patients, respectively $(\mathrm{P}=0.004)$. However, we did not observe a significant difference in LEF1 expression between CD38-positive and CD38-negative patients. Moreover, patients requiring treatment showed a mean LEF1 RER of 85.61 whereas patients in recently diagnosed Binet A stage had a mean of only 22.01 $(\mathrm{P}<0.001)$. We also found significant correlations of LEF1 with the percentage of lymphocytes and FMOD expression. Our results suggest that high LEF1 expression is associated with poor prognosis and disease progression. Thus, LEF1 might be involved in the process of disease progression and possibly can serve as a molecular parameter for risk assessment and/or monitoring of CLL.
\end{abstract}

\section{Introduction}

Chronic lymphocytic leukemia (CLL) is the most common leukemia in the Western world. It is characterized by accumulation of monoclonal $\mathrm{CD}^{+} \mathrm{B}$-lymphocytes and has a highly variable clinical outcome. Lymphoid enhancer-binding factor-1 (LEF1) is part of the LEF/TCF transcription factor family which has been shown to play an important role in regulating Wnt-pathway target genes. ${ }^{1,2}$ LEF1 is specifically expressed at early stages of B-cell differentiation and is essential for survival and proliferation. ${ }^{3}$

LEF1 plays a crucial role in many human cancers. ${ }^{4-6}$ It has been shown to be overexpressed in CLL. ${ }^{78}$ However, the extent of over- expression shows a high variability between different patients. Previous research suggests a possible link between the degree of LEF1 overexpression and poor prognosis in leukemic diseases. For example, Wang et al. showed that LEF1 expression in highly malignant acute leukemias is higher than in lowgrade chronic leukemias. ${ }^{4}$ Moreover, in acute lymphoblastic leukemia, patients with higher LEF1 mRNA expression caused by epigenetic silencing of Wnt-suppressors have an even worse prognosis compared to patients with relatively lower LEF1 expression. ${ }^{9}$

The aim of this study was to show the prognostic relevance of LEF1 expression in CLL. We assessed correlations of LEF1 with the zetaassociated protein 70 (ZAP70) and CD38. Both parameters have been shown to be useful prognostic markers in CLL. ${ }^{10,11}$ Moreover, we compared LEF1 expression of patients in Binet C stage and patients requiring treatment in Binet $\mathrm{A} / \mathrm{B}$ stage with the LEF1 expression of patients in recently diagnosed Binet A stage. We further investigated the association between LEF1 and fibromodulin (FMOD). FMOD is a known tumor-associated antigen in CLL. ${ }^{7,8}$ Vallat et al. showed that fibromodulin mRNA is highly expressed in p53-mutated CLL cells. ${ }^{12}$ Mutations of p53 are known to be associated with poor prognosis and therapy resistance in CLL. ${ }^{13,14}$

\section{Materials and Methods}

One hundred and twelve anonymized CLL patient samples left over from routine diagnostic tests were enriched using RossetteSep ${ }^{\circledR}$ (StemCell Technologies, Vancouver, BC, Canada). The study includes patients of all Binet stages, untreated patients, and patients with a maximum of up to three prior treatment regimens. Fifty-four patient samples were directly processed. Fifty-eight additional samples were first frozen at $-80^{\circ} \mathrm{C}$ in RPMI 1640 media containing $20 \%$ fetal cow serum (Biochrome AG, Berlin, Germany) and 10\% dimethyl sulfoxide (Roth, Karlsruhe, Germany) and later processed. Fresh and frozen samples matched for Binet stage did not differ significantly in LEF1 mRNA expression. B-cells of seven anonymized samples left over from healthy blood donors were used as healthy controls. Four healthy samples were enriched using CD19 microbeats (Miltenyi Biotech, Bergisch Gladbach, Germany). The other three healthy samples were purified using Rossette $\mathrm{Sep}^{\circledR}$. The healthy samples processed with the two different methods did not differ significantly in LEF1 mRNA expression.

Purification of mRNA was performed using QIAamp RNA Blood Mini Kit (Qiagen, Hilden, Germany). For reverse transcription to cDNA,
Correspondence: Karl-Anton Kreuzer, Department I of Internal Medicine, University at Cologne, Kerpener Strasse 62, 50937 Cologne, Germany. E-mail: karl-anton.kreuzer@uni-koeln.de

Key words: lymphoid enhancer-binding factor-1 (LEF1), chronic lymphocytic leukemia (CLL), zeta-associated protein (ZAP70), CD38, fibromodulin.

Received for publication: 24 February 2010.

Revision received: 19 April 2010.

Accepted for publication: 22 April 2010.

This work is licensed under a Creative Commons Attribution 3.0 License (by-nc 3.0).

(C) Copyright F. Erdfelder et al., 2010

Licensee PAGEPress, Italy

Hematology Reports 2010; 2:e3

doi:10.4081/hr.2010.e3

SuperScript $^{\circledR}$ III First-Strand Synthesis System for RT-PCR (Invitrogen, Karlsruhe, Germany) was used. TaqMan ${ }^{\circledR}$ Probe real-time PCR was performed using LightCycler ${ }^{\circledR}$ FastStart DNA MasterPLUS HybProbe Kit (Roche, GrenzachWyhlen, Germany). All PCR assays were performed in a LightCycler ${ }^{\circledR} 2.0$ Instrument. All assays were started with a denaturation program $\left(40^{\circ} \mathrm{C}\right.$ for $5 \mathrm{~min}, 95^{\circ} \mathrm{C}$ for $5 \mathrm{~min}$ ) followed by an amplification and quantification program with a single fluorescence measurement repeated 45 times. The amplification and quantification program was individually optimized for each assay. All assays were ended with a cooling step to $40^{\circ} \mathrm{C}$. For LEF1-PCR, the forward primer 5'-GCCACGGACGAGATGATCC3 , the reverse primer 5'-TGTCTGGCCACCTCGTGTC-3', and the probe 5'-6FAM-TCAAGGACGAGGGCGATCCTCAGAAGGAA-Dabcyl-3' were used. The amplification and quantification program for LEF1-PCR was $95^{\circ} \mathrm{C}$ for $10 \mathrm{~s}$, $62^{\circ} \mathrm{C}$ for $15 \mathrm{~s}$, and $72^{\circ} \mathrm{C}$ for $15 \mathrm{~s}$. For FMODPCR, the forward primer 5'ATGACCCTCATTGGTGGTTCC-3', the reverse primer 5'-GGAGGTGATCTGGTTGTTCTGGA-3', and the probe 5'6FAM-TACGGCTCTCCATCCCCTCCAGATCCCCGCGACT-TMR-3' were used. The amplification and quantification program for FMODPCR was $95^{\circ} \mathrm{C}$ for $10 \mathrm{~s}$ and $65^{\circ} \mathrm{C}$ for $20 \mathrm{~s}$. FMOD RER could be determined for only 96 samples because of insufficient sample volume. ABL was used as the housekeeping gene with the forward primer 5'-TGGAGATAACACTCTAAGCATAACTAAAGGT-3', the reverse primer 5'GATGATGTTGCTTGGGACCCA-3', and the probe 5'-6FAM-CCATTTTTGGTTTGGGCTTCACACCATT-Dabcyl-3'. The amplification and quantification program for ABL-PCR was $95^{\circ} \mathrm{C}$ for $10 \mathrm{~s}, 60^{\circ} \mathrm{C}$ for $15 \mathrm{~s}$, and $72^{\circ} \mathrm{C}$ for $15 \mathrm{~s}$. All primers and probes were purchased from TIB MOLBIOL (Berlin, Germany). Cycle thresholds were determined by the second derivative 
maximum algorithm using LightCycler ${ }^{\circledR}$ Software 3.5 (Roche, Grenzach-Wyhlen, Germany). PCR-efficiency-corrected calibrator-normalized relative expression ratios (RER) were calculated according to Roche Applied Science Technical Note No. LC $13 / 2001 .^{15}$ The healthy B-cell samples were used for calibration. In other words, the calibrated mean RER of healthy B-cells is 1 by definition, and all other RER values are expressed as fold change compared to healthy B-cells. PCR-efficiency was calculated according to the equation: $E=10^{\wedge}(-1 /$ slope $) .{ }^{16}$ The dilution series showed high linearity (Pearson correlation coefficient $r>0.99$ ) for all RT-PCR assays. All sample processing steps were performed according to the respective manufacturer's protocol.

Immunophenotypic measurements (CD38, ZAP70, lymphocyte percentage) were performed by a routine diagnostic laboratory using flow cytometry. The ZAP70 assay was performed according to the method of Rassenti et al. ${ }^{10}$ using a monoclonal antibody specific for ZAP70 (clone 1E7.2) conjugated with Alexa Flour 488 (Invitrogen, Karlsruhe, Germany). For the CD38 assay, the monoclonal antibody A07780 (Clone LS198-4-3) labeled with R Phycoerythrin-Cyanin 5.1 (Beckmann Coulter, Krefeld, Germany) was used.

Statistical analyses were performed using GraphPad Prism 4 (GraphPad Software, La Jolla, CA, USA) and the SPSS 17 software (SPSS, Chicago, IL, USA). Because the assumptions underlying the General Linear Model were obviously violated for our data, we used nonparametric statistical methods such as the Mann-Whitney U test and both Kendall's and Spearman's rank correlation coefficients.

\section{Results}

Our correlation analyses revealed significantly positive Spearman and Kendall rank correlations of LEF1 with the percentage of ZAP70-positive CLL cells. Moreover, LEF1 was also positively correlated with the percentage of lymphocytes in the peripheral blood and with the FMOD mRNA expression. However, we did not observe a positive correlation of LEF1 with CD38 (Table 1).

Patients showing ZAP70 expression in at least $20 \%$ of the CLL cells were labeled ZAP70 positive. ${ }^{10}$ Similarly, patients showing CD38 expression in at least $30 \%$ of the CLL cells were classified as CD38 positive. ${ }^{11}$ Comparing ZAP70-positive and -negative patients, we found mean LEF1 RERs of 53.72 and 37.10 in the positive and the negative group, respectively. This difference is highly significant (Mann Whitney $\mathrm{U}=866.50, \mathrm{P}=0.004$ ) (Figure 1A). Replicating the results of our correlation
Table 1. Kendall's and Spearman's rank correlation coefficients between LEF1 and ZAP70, percentage of lymphocytes in the peripheral blood (\% Lymph), CD38, and FMOD.

\begin{tabular}{llcccc}
\hline \multirow{2}{*}{ Kendall's } & ZAP70 & \% Lymph & CD38 & FMOD \\
& Tau correlation & 0.203 & 0.296 & -0.100 & 0.302 \\
& coefficient & & & & \\
& $\mathrm{P}$ & 0.002 & $<0.001$ & 0.119 & $<0.001$ \\
Spearman's & $\mathrm{n}$ & 112 & 112 & 112 & 96 \\
& Rho correlation & 0.300 & 0.440 & -0.152 & 0.450 \\
& coefficient & & & & \\
& $\mathrm{P}$ & 0.001 & $<0.001$ & 0.111 & $<0.001$ \\
& $\mathrm{n}$ & 112 & 112 & 112 & 96 \\
\hline
\end{tabular}
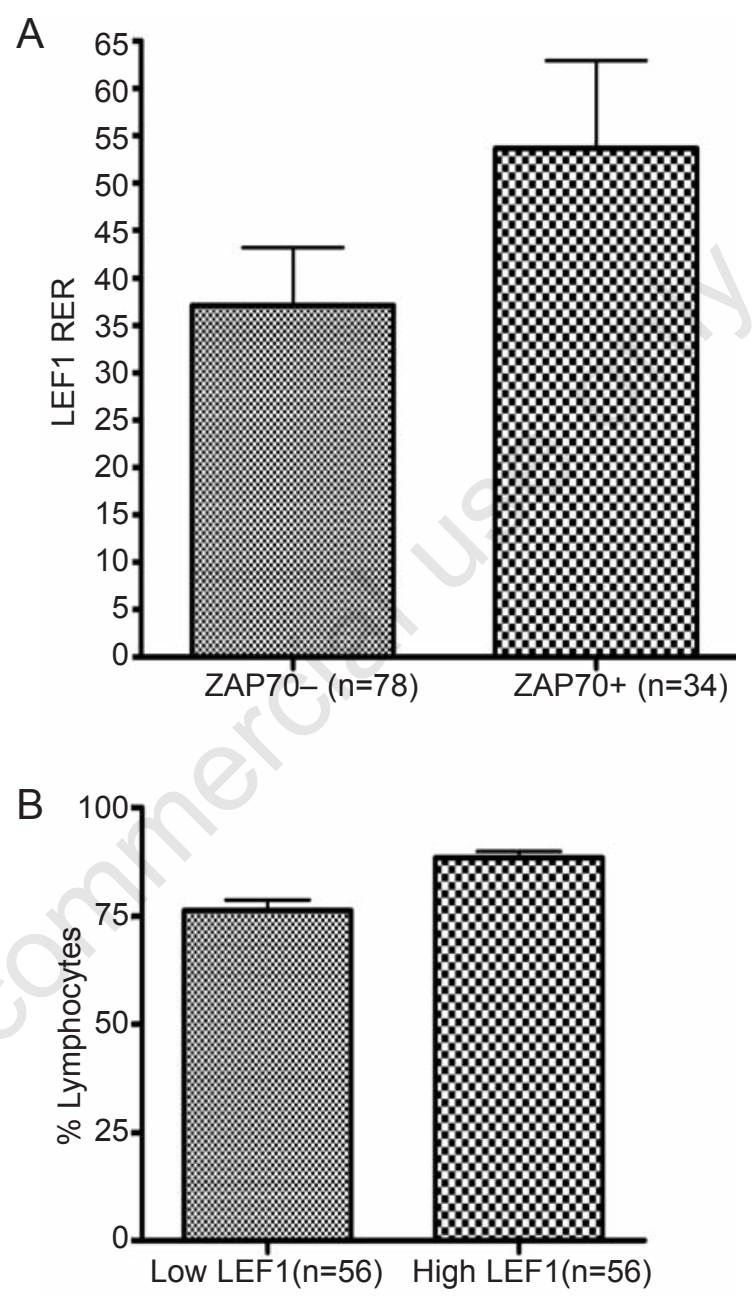

Figure 1. (A) Mean LEF1
RER (and standard errors)
for ZAP70-positive and
-negative patients (Mann-
Whitney U=866.50,
P=0.004). (B) Mean per-
centage of lymphocytes in
the peripheral blood (and
standard errors) for high
and low LEF1 samples
(high = above LEF1 me-
dian, low = under LEF1
median) (Mann-Whitney
$U=828.50, P<0.001$ ).

analyses, we did not observe a significant difference in CD38-positive and -negative patients (data not shown).

We performed a LEF1 RER median split dividing the patients in high and low LEF1 subgroups. We then compared the percentages of lymphocytes in the peripheral blood and the FMOD mRNA expressions in both subgroups. We observed mean lymphocyte percentages of $88.45 \%$ and $76.28 \%$ in the high versus low LEF1 groups, respectively (Mann-Whitney $\mathrm{U}=828.50, \mathrm{P}<0.001$ ) (Figure $1 \mathrm{~B})$. The mean FMOD RER was 34.14 in the high LEF1 group and only 4.19 in the low LEF1 group (MannWhitney $\mathrm{U}=554.00, \mathrm{P}<0.001$ ) (Figure $2 \mathrm{~A}$ ).
The mean LEF1 mRNA expression in our CLL patients' samples was 42-fold higher compared to the healthy B-cell samples (Figure 2B). This difference was highly significant (Mann-Whitney $\mathrm{U}=32.00, \mathrm{P}<0.001$ ).

Patients without pretreatment were split in two groups. Group 1 consisted of Binet A stage patients diagnosed less than one year ago. Group 2 included patients in stage Binet $\mathrm{C}$ and Binet $\mathrm{A} / \mathrm{B}$ patients requiring treatment. The patients in the first group showed a mean LEF1 RER of 22.01 whereas those of the second group showed a mean of 85.61. This difference was also highly significant (Mann-Whitney $\mathrm{U}=341.50, \mathrm{P}<0.001$ ) (Figure 3 ). 


\section{Discussion}

Our finding that LEF1 is overexpressed in CLL cells compared to healthy B-cells is consistent with the results of previous studies. ${ }^{7,8}$ In addition, we found that LEF1 expression in CLL patients is associated with ZAP70 positivity, high percentages of lymphocytes in the peripheral blood, and CLL disease stages requiring treatment. This suggests a significant role of LEF1 with respect to prognosis and disease progression in CLL. In contrast, we found no correlation between LEF1 and CD38. CD38 has been shown previously to be an IgVH-mutation-status independent prognostic factor. ${ }^{11}$ Hence, one possible interpretation of our results is that the prognostic subgroup defined by CD38 positivity is also LEF1 independent. Another possible explanation could be CD38 variability during the course of CLL, ${ }^{11}$ which reduces the LEF1-CD38 correlation. We were also able to show a strong association between LEF1 and FMOD mRNA expression in CLL. Both proteins have been shown to be tumor-associated antigens in CLL. ${ }^{7,8}$ Given the fact that FMOD expression, p53 mutation, and DNA-damage resistance have been found previously to be associated, ${ }^{12}$ the LEF1-FMOD correlation is an interesting new finding. Eventually, it may lend additional support to the prognostic relevance of LEF1 overexpression in CLL.

In addition to its prognostic relevance, LEF1 is probably of great functional importance. Two lines of evidence suggest a possible causal role of LEF1 in CLL pathogenesis. First, several studies found effects of LEF1 expression on the degree of malignancy in neoplastic diseases. For example, LEF1 appears to mediate tumor growth and invasion ability in androgen-independent prostate cancer. ${ }^{5}$ Moreover, Nguyen $e t$ al. found that LEF1 also mediates cell invasion in breast cancer. ${ }^{6}$ Second, deregulated LEF1 expression may also be an important step in the development of neoplastic diseases. Consistent with this idea, Jelinek et al. hypothesized a connection between deregulated LEF1 expression and clonal expansion of Bcells in CLL. ${ }^{7}$ Other studies lend additional support to the hypothesis of LEF1 overexpression being an important oncogenic factor. For example, LEF1 has been shown to reduce or even eliminate transforming growth factor $\beta$ mediated repression of the $c$-myc oncogene. ${ }^{17}$ Furthermore, Rivat et al. found LEF1 to take part in transactivating the matrix metalloprotease matrilysin, which plays a key role in local invasion of digestive tumors. ${ }^{18}$ In addition, neoplastic transformation induced by LEF1 has been shown both in vitro and in vivo. For example, Aoki et al. provided evidence for an oncogenic effect of LEF1 in chicken fibroblasts in vitro. ${ }^{19}$ Moreover, deregulated expression of

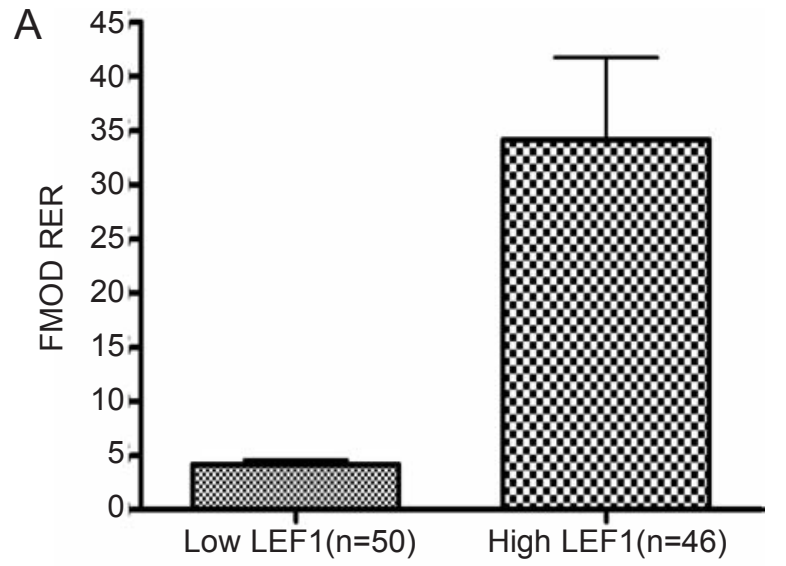

Figure 2. (A) Mean FMOD RER (and standard errors) for high and low LEF1 samples (high $=$ above LEF1 median, low $=$ under LEF1 median) (MannWhitney $U=554.00$, P<0.001). (B) Scatter plot of LEF1 RER (and means) for healthy B-cells and CLL samples (MannWhitney $U=32.00$, $\mathbf{P}<0.001)$. Each of the 112 CLL and 7 healthy B-cell samples is represented a doi.
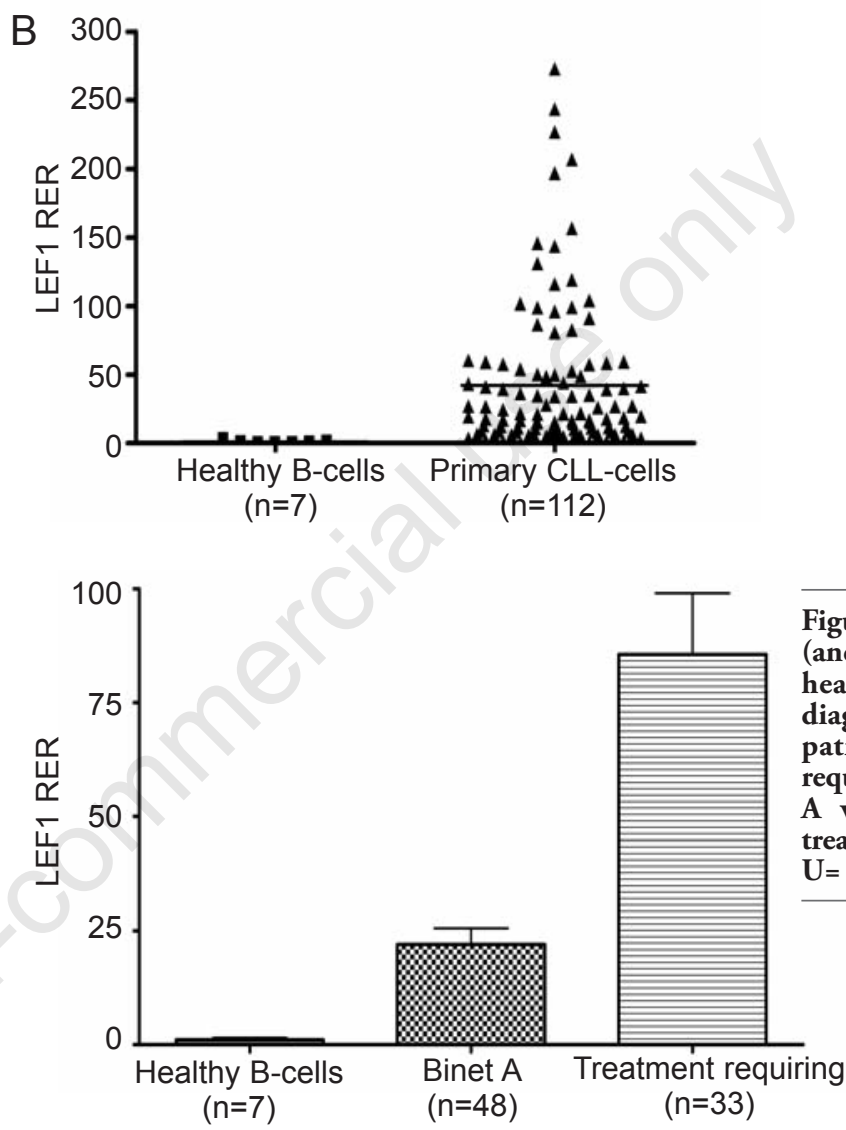

LEF1 in vivo has been shown to induce acute B-lymphoblastic leukemias and acute myeloid leukemias in mice. ${ }^{20}$

\section{Conclusions}

In a nutshell, our results suggest that high LEF1 expression is associated with poor prognosis and disease progression in CLL. Thus, LEF1 might be involved in the process of disease progression, and possibly can serve as a molecular parameter for risk assessment and/or monitoring of CLL. Studies investigating LEF1 as a possible therapeutic target in CLL are underway.

\section{References}

1. Behrens J, von Kries JP, Kuhl M, et al. Functional interaction of beta-catenin with the transcription factor LEF-1. Nature 1996;382:638-42.

2. Huber 0, Korn R, McLaughlin J, et al. Nuclear localization of beta-catenin by interaction with transcription factor LEF1. Mech Dev 1996;59:3-10.

3. Reya T, O'Riordan M, Okamura R, et al. Wnt signaling regulates $\mathrm{B}$ lymphocyte proliferation through a LEF-1 dependent mechanism. Immunity 2000;13:15-24.

4. Wang W, Ji P, Steffen B, et al. Alterations of lymphoid enhancer factor-1 isoform 
expression in solid tumors and acute leukemias. Acta Biochim Biophys Sin (Shanghai) 2005;37:173-80.

5. Li Y, Wang L, Zhang M, et al. LEF1 in androgen-independent prostate cancer: regulation of androgen receptor expression, prostate cancer growth, and invasion. Cancer Res 2009;69:3332-8.

6. Nguyen A, Rosner A, Milovanovic T, et al. Wnt pathway component LEF1 mediates tumor cell invasion and is expressed in human and murine breast cancers lacking ErbB2 (her-2/neu) overexpression. Int $\mathbf{J}$ Oncol 2005;27:949-56.

7. Jelinek DF, Tschumper RC, Stolovitzky GA, et al. Identification of a global gene expression signature of B-chronic lymphocytic leukemia. Mol Cancer Res 2003;1: 346-61.

8. Klein U, Tu Y, Stolovitzky GA, et al. Gene expression profiling of B cell chronic lymphocytic leukemia reveals a homogeneous phenotype related to memory B cells. J Exp Med 2001;194:1625-38.

9. Roman-Gomez J, Cordeu L, Agirre X, et al. Epigenetic regulation of Wnt-signaling pathway in acute lymphoblastic leukemia. Blood 2007;109:3462-9.
10. Rassenti LZ, Huynh L, Toy TL, et al. ZAP-70 compared with immunoglobulin heavychain gene mutation status as a predictor of disease progression in chronic lymphocytic leukemia. N Engl J Med 2004;351: 893-901.

11. Hamblin TJ, Orchard JA, Ibbotson RE, et al. CD38 expression and immunoglobulin variable region mutations are independent prognostic variables in chronic lymphocytic leukemia, but CD38 expression may vary during the course of the disease. Blood 2002;99:1023-9.

12. Vallat L, Magdelenat H, Merle-Beral H, et al. The resistance of B-CLL cells to DNA damage-induced apoptosis defined by DNA microarrays. Blood 2003;101:4598-606.

13. Zenz T, Benner A, Dohner H, et al. Chronic lymphocytic leukemia and treatment resistance in cancer: the role of the p53 pathway. Cell Cycle 2008;7:3810-4.

14. Malcikova J, Smardova J, Rocnova L, et al. Monoallelic and biallelic inactivation of TP53 gene in chronic lymphocytic leukemia: selection, impact on survival, and response to DNA damage. Blood 2009; 114:5307-14.

15. Roche Applied Science Technical Note No.
LC 13/2001 (31), 4. Calibrator Normalized Relative Quantification, http://www.genequantification.de/roche-rel-quant.pdf

16. Rasmussen R. Quantification on the Light Cycler. In: rapid cycle real-time PCR, methods and applications. Meuer S, Wittwer C, Nakagawara K, Eds. Heidelberg: Springer Press, 2000, pp 21-34.

17. Sasaki T, Suzuki H, Yagi K, et al. Lymphoid enhancer factor 1 makes cells resistant to transforming growth factor beta-induced repression of c-myc. Cancer Res 2003;63: 801-6.

18. Rivat C, Le FN, Sabbah M, et al. Synergistic cooperation between the AP-1 and LEF-1 transcription factors in activation of the matrilysin promoter by the src oncogene: implications in cellular invasion. FASEB $\mathrm{J}$ 2003;17:1721-3.

19. Aoki M, Hecht A, Kruse U, et al. Nuclear endpoint of Wnt signaling: neoplastic transformation induced by transactivating lymphoid-enhancing factor 1. Proc Natl Acad Sci USA 1999;96:139-44.

20. Petropoulos K, Arseni N, Schessl C, et al. A novel role for Lef-1, a central transcription mediator of Wnt signaling, in leukemogenesis. J Exp Med 2008;205:515-22. 